\title{
Ceftriaxone Induced Unilateral Facial Angioedema
}

\author{
Recep Alanli ${ }^{1}$
}

${ }^{1}$ Department of Internal Medicine, Lokman Hekim Ankara Hospital, Lokman Hekim University, Ankara, Turkey

\section{Correspondence:}

Recep Alanli, MD

Address: Department of Internal Medicine, Lokman Hekim Ankara Hospital, Lokman Hekim University, Ankara, Turkey

Email: recepalanli@gmail.com
Received: 11.02.2019,

Accepted: 19.02.2019

https://doi.org/10.5799/jcei/5760

\section{INTRODUCTION}

Angioedema is a transient and life threatening swelling of mucosa and submucosa. Angioedema generally develops over minutes to hours and resolves in 24 to 48 hours. It can occur in periorbital region, lips, tongue, extremities and bowel wall. The most common sites for angioedema are periorbital region and lips. Angioedema can be mediated by histamine, bradykinin, or other mechanism. Angioedema is categorized into allergic and nonallergic forms. Allergic angioedema occurs due to the release of histamines, leukotrienes, and prostaglandins from mast cells. These mediators cause transient increase in the permeability of postcapillary venules and fluid leakage in subcutaneous and submucosal tissues [1]. Allergic forms are precipitated by foods or allergens, and mediated by IgE. Fresh fruits, fish, milk products, chocolate, peanuts, pollens, and drugs are considered as major allergens. Common drugs that cause angioedema are; nonsteroidal antiinflamatory drugs (NSAIDs), diuretics, and penicillin. Nonallergic forms include angiotensinconverting enzyme (ACE) inhibitor induced, hereditary, and acquired angioedema. These types are thought to be related to bradykinin [2], a potent vasodilator and mediator of vascular permeability. ACE inhibitor, catalyses the breakdown of serum bradykinin and substance $P$. These mediators cause vasodilatation and increased capillary permeability leading to angioedema. Hereditary angioedema is a rare autosomal dominant condition due to deficiency or dysfunction of $\mathrm{C} 1$ esterase inhibitor protein. This causes massive activation of the complement system and increases bradykinin levels. Acquired forms are associated with lymphoproliferative and autoimmune diseases. Physical factors which causes angioedema are cold, heat, mechanical irritation, exercise, emotional stress, and ultraviolet light [3]. Idiopathic angioedema is considered when no causative factors can be identified. Identification of the etiologic factor and its elimination is the most important factor in treatment. Airway must be secured while initiating specific treatment. To ensure that patients are managed correctly, identification of the underlying cause of angioedema on presentation is essential. Distinguishing histamine- versus bradykinin- mediated angioedema is important. Urticaria and itching are common in histamine mediated angioedema. Bradykinin mediated attacks are more likely to have abdominal pain. A low serum C4 level is a good screening test for hereditary angioedema. Histamine mediated angioedema should be treated with $\mathrm{H} 1$ and $\mathrm{H} 2$ antagonists and systemic glucocorticoids along with epinephrine, as appropriate. Bradykinin mediated angioedema won't respond to this treatment and may be treated with fresh frozen plasma. 
Hereditary angioedema should be treated with C1-INH inhibitor. Monitoring the patient for at least 24 hours is mandatory in angioedema [4].

\section{CASE REPORT}

A 61-year-old Caucaussian male patient was referred to general surgery service with chief complaints of nausea, vomiting, and abdominal pain because of acute cholecystitis. His past medical history included; hypertension, diabetes mellitus, hyperlipidemia, chronic hepatitis $\mathrm{B}$, and cerebrovascular disease. He was taking metformin, acetyl salicylic acid, fenofibrate, valsartan and hydrochlorothiazide. He had no known drug allergies or operation history. Abdominal ultrasound revealed thickened gallbladder wall, with pericholecystic fluid and a 10x8mm sized calculus. Significant laboratory results were white blood count (WBC) of $17000 \mathrm{~mm} 3$ and CRP $214 \mathrm{mg} / \mathrm{dl}$. Other results were; ALT $18 \mathrm{U} / \mathrm{L}$, AST 15U/L ALP $49 \mathrm{U} / \mathrm{L}$, GGT 29U/L and total biluribin $0,7 \mathrm{mg} / \mathrm{dl}$. These findings were suggesting acute cholcystitis. Diclofenac sodium and ceftriaxone treatment was started for acute cholecystitis. The patient's condition improved after treatment and he refused the operation. Two days after the patient's admission to the surgery clinic, he developed a sudden right sided facial swelling involving tongue and lip and was diagnosed as angioedema. Angioedema developed 8 hours after injection of ceftriaxone (1000mg) and 15 minutes after diclofenac sodium (75mg). There was no history of foreign body ingestion or trauma to the tongue or neck. On physical examination, right side of lips and tongue were significantly swollen without tenderness. There was no mandibular tenderness or cervical lymphadenopathy. Vital signs were; blood pressure as 143/77 $\mathrm{mmHg}$, pulses as 98 per minute, respiratory rate as 22 per minute and oxygen saturation of $97 \%$ on room air. He had odynophagia, hoarseness and shortness of breath. There was no urticaria on body or extremities. Other signs were unremarkable. The patient's CRP was $101 \mathrm{mg} / \mathrm{dl}$ but other laboratory results were within normal limits. Diclofenac sodium was discontinued after angioedema. The patient was treated with intravenous dexamethasone, famotidine and diphenhydramine. He was transferred to the intensive care unit because of angioedema for further monitoring. Three hours later, swelling on lips and tongue had reduced significantly. The patient was consulted to an ENT specialist for airway evaluation. Laryngoscopy demonstrated angioedema of the right base of tongue not extending to the vallecula and epiglottis. After a thorough neurological examination and MRI scan, no neurological pathology was reported. Complement 3 (C3) level was $135 \mathrm{mg} / \mathrm{dL}$ and complement 4 (C4) level was $13 \mathrm{mg} / \mathrm{dL}$, which were normal. Two days later, 10 minutes after ceftriaxone administration, left sided facial angioedema developed. His physical examination was similar with prior angioedema attack. The patient was treated with intravenous dexamethasone, famotidine and antihistaminic again. His condition improved and three days later he was discharged home.

\section{DISCUSSION}

Unilateral angioedema is uncommon. The exact mechanism is not known. Although there are many causes of angioedema, most agree that there are four main causes: allergic reaction to food, drug, or environmental exposure due to an Ig E-depended reaction, congenital angioedema, ACE inhibitors and idiopathic [5].

Diclofenac sodium is a NSAID, and commonly used to treat fever, inflammation, and pain. NSAIDs are the most common prescribed class of drug in the world. Also they are the most common cause of all adverse drug reactions. Rhinoconjuctivitis, bronchospasm, urticaria, angioedema, and anaphylaxis can occur as an allergic reaction to NSAIDs. The prevalence of urticaria and angioedema to NSAIDs has been reported to be $0.1 \%$ to $3 \%$ worldwide [6]. Leeyapan et al. [7] reported that $50 \%$ of patients with drug induced angioedema resulted from NSAIDs. In an Italian review of 2137 reactions, NSAIDs were causative agents in $33.6 \%$ of patients for drug-induced angioedema [8]. First attack of angioedema on this reported patient was after 15 minutes of diclofenac administration and for the reason diclofenac was thought to be causative agent.

In one study oral angioedema was reported after 10 minutes of ceftriaxone administration [9]. The second angioedema attack of the reported patient was seen after discontinuation of diclofenac and after 10 minutes of ceftriaxone administration, therefore ceftriaxone was thought to be the causative agent. To our knowledge there is no previous report about ceftriaxone induced unilateral angioedema. This can be the first report about ceftriaxone induced unilateral angioedema.

Hereditary angioedema patients have family history, they do not experience pruritus or urticarial lesions and complement factors C2 and C4 levels are low. For this reported patient $\mathrm{C} 4$ level was normal and had no family history. So hereditary angioedema was ruled out.

The reported incidence of angioedema secondary to ACE inhibitors ranges from 0.1 to $0.7 \%$ [10]. Angiotensin II receptor blockers (A2RB) do not affect bradykinin degradation. However, a meta analysis suggests that patients who develope angioedema after ACE inhibitors have a risk of developing angioedema after A2RB usage up to $9 \%$. Incidence is highest in the first month of drug usage, but angioedema can occur even after years of usage [11]. This reported patient has $\mathrm{A} 2 \mathrm{RB}$ usage history but he had no history of ACE inhibitor induced angioedema.

In one study aspirin intolerance manifest as unilateral left periorbital edema [12]. In this reported patient aspirin was discontinued while he admitted to hospital because of the planned operation. There is no evidence for aspirin induced angioedema for this reported patient. 


\section{CONCLUSION}

Unilateral facial angioedema after ceftriaxone usage is a very rare event that has not been reported earlier and must be considered if there are no other cause determined.

Declaration of interest: The author report no conflicts of interest.

Financial Disclosure: No financial support was received.

\section{REFERENCES}

1. Bas M, Adams V, Suvorava $\mathrm{T}$, et al. Unusual radiographic appearance of drug-induced pharyngeal angioedema and differential considerations. AJNR Am J Neuroradiol. 2009;30(1):77-8.

2. Conlin JL, Mian AZ, Sakai O. Unilateral mass-like pharyngeal and laryngeal angioedema, Emerg Radiol. 2011;18:441-3.

3. Temino VM, Peebles RS Jr. The spectrum and treatment of angioedema, Am J Med. 2008;121:282-6.

4. Bernstein JA, Cremonesi P, Hoffmann TK, Hollingsworth J. Angioedema in the emergency department: a practical guide to differential diagnosis and management: Int J Emerg Med. 2017;10:15.

5. Zirkle M, Bhattacharyya N. Predictors airway intervention in angioedema of the head and neck. Otolaryngol Head Neck Surg. 2000;123:240-5.

6. Kulthanan K, Jiamton S, Boochangkool K, et al. Angioedema: clinical and etiological aspects. Clin Dev Immunol. 2007;2007:26438.
7. Leeyaphan C, Kulthanan K, Jongiarearnprasert K, et al. Drug-induced angioedema without urticaria: prevalence and clinical features. J Eur Acad Dermatol Venerol. 2010;24:685-91.

8. Cutaneous reactions to analgesic-antipyretics and nonsteroidal anti-inflammatory drugs. Analysis of reports to the spontaneous reporting system of the Gruppo Italiano Studi Epidemiologici in Dermatologia. Dermatology. 1993;186:164-9.

9. Poston SA, Jennings HR, Poe KL. Cefazolin tolerance does not predict ceftriaxone hypersensitivity: unique side chains precipitate anaphylaxis. Pharmacotherapy. 2004;24(5):668-72.

10. Nazaneen NG, Ziad ED, Stanley HC. Clinical experience with angiotensin-converting enzyme inhibitor-induced angioedema. Otolaryngol Head Neck Surg. 2007;137:931-5.

11. Haymore BR, Yoon J, Mitika CP, et al. Risk of angioedema with angiotensin receptor blockers in patients with prior angioedema associated with angiotensin-converting enzyme inhibitors: a metaanalysis. Ann Allergy Asthma Immunol. 2008;101:4959.

12. Kurtseen S Price, MD and David M P Thomson. Localized unilateral periorbital edema induced by aspirin, Ann Allergy Asthma Immunol. 1997;79:42022.33 . 